\begin{tabular}{|c|l|}
\hline Title & Mean-field study of correlation-induced antisymmetric spin-orbit coupling in a two-orbital honey comb model \\
\hline Author(s) & Hayami, Satoru; Kusunose, Hiroaki; Motome, Y ukitoshi \\
\hline Citation & $\begin{array}{l}\text { Physica B : Condensed Matter, 536, 649-653 } \\
\text { https://doi.org/_0.1016/.physb.2017.08.063 }\end{array}$ \\
\hline Issue Date & 2018-05-01 \\
\hline Doc URL & http://hdl.handle.net/2115/77802 \\
\hline Rights & $\begin{array}{l}\text { O2018. This manuscript version is made available under the CC-BY-NC-ND 4.0 license } \\
\text { http://creativecommons.org/icenses/by-nc-nd/4.0/ }\end{array}$ \\
\hline Rights(URL) & http://creativecommons.org/icenses/by-nc-nd/4.0/ \\
\hline Type & article (author version) \\
\hline hile Information & honeycomb_MF_submit_Huscap.pdf \\
\hline
\end{tabular}

Instructions for use 


\title{
Mean-field study of correlation-induced antisymmetric spin-orbit coupling in a two-orbital honeycomb model
}

\author{
Satoru Hayami ${ }^{1}$, Hiroaki Kusunose ${ }^{2}$, and Yukitoshi Motome ${ }^{3}$ \\ 1 Department of Physics, Hokkaido University, Sapporo 060-0810, Japan \\ 2 Department of Physics, Meiji University, Kawasaki 214-8571, Japan \\ 3 Department of Applied Physics, University of Tokyo, Tokyo 113-8656, Japan
}

\begin{abstract}
We investigate a two-orbital Hubbard model on a honeycomb structure, with a special focus on the antisymmetric spin-orbit coupling (ASOC) induced by symmetry breaking in the electronic degrees of freedom. By investigating the ground-state phase diagram by the mean-field approximation in addition to the analysis in the strong correlation limit, we obtain a variety of symmetrybroken phases that induce different types of effective ASOCs by breaking of spatial inversion symmetry. We find several unusual properties emergent from the ASOCs, such as a linear magnetoelectric effect in a spin-orbital ordered phase at $1 / 4$ filling and a spin splitting in the band structure in charge ordered phases at $1 / 4$ and $1 / 2$ fillings. We also show that a staggered potential on the honeycomb structure leads to another type of ASOC, which gives rise to a valley splitting in the band structure at $1 / 2$ filling. We discuss the experimental relevance of our results to candidate materials including transition metal dichalcogenides and trichalcogenides.
\end{abstract}

Keywords: spin-orbit coupling; multipole; spatial inversion symmetry breaking; spin and valley splittings; magnetoelectric effect; transition metal dichalcogenides and trichalcogenides

Email address: hayami@phys.sci.hokudai.ac.jp (Satoru Hayami ${ }^{1}$ ) 


\section{Introduction}

The relativistic spin-orbit coupling (SOC) in solids can acquire a particular spin and wave-number dependence when the system breaks spatial inversion symmetry. This is called the antisymmetric SOC (ASOC), which has recently

5 attracted much attention in condensed matter physics, such as noncentrosymmetric superconductors, topological insulators, and multiferroics [1, 2, 3, 4]. The peculiar spin and wave-number dependence leads to fascinating electronic properties, such as unconventional superconductivities, the spin Hall effect, and magnetoelectric effects $[1,5,6,7]$. Such interesting phenomena associated with the ASOC have drawn considerable attention for potential applications to electronics and spintronics devices.

Recently, the possibility of ASOCs on a centrosymmetric lattice structure has been explored, such as on a zigzag chain, a honeycomb structure, and a triangular lattice $[8,9,10,11,12,13,14,15,16]$. In these situations, the ASOC is effectively induced by the spontaneous formation of an electronic order with spatial inversion symmetry breaking. Such emergent ASOCs are intriguing as they result in the asymmetry in the electronic structures $[17,18,8,9,13]$, spin-wave excitations [19, 20, 21], and nonreciprocal optical responses [22]. Furthermore, these types of ASOC are more flexibly controlled than those in noncentrosymmetric systems: in stark contrast to the latter usually fixed by the lattice and electronic structures, the ASOCs induced by electron correlations are controllable in their spin and wave-number dependence, even switched on and off, by external parameters, such as temperature and pressure. Thus, such correlation-induced ASOCs are expected to bring about new spin-orbital entangled phenomena.

In the previous studies $[11,23,15]$, the authors have demonstrated how electronic orderings can induce the effective ASOCs, taking a fundamental model, a two-orbital Hubbard model defined on a honeycomb structure. Through these studies, the nature of the correlation-induced ASOCs were elucidated by the symmetry analysis and the linear response theory. It was shown that the emer- 
gent ASOCs are a source of peculiar properties, such as the spin and valley splittings in the band structure and unconventional cross correlations among spin, charge, and orbital degrees of freedom. However, the stability of the symmetry-broken phases and the phase transitions were not fully investigated.

35 It is desired to systematically study the phase diagram for clarifying when and how the effective ASOCs are activated.

In the present study, we investigate what types of electronic orderings are realized in the ground state for the two-orbital model on the honeycomb structure [11]. By the mean-field approximation, we construct the ground-state phase diagram at two commensurate electron fillings, $1 / 4$ and $1 / 2$ fillings. We find several different types of ordered phases that break spatial inversion symmetry by staggered-type electronic ordering: for instance, a spin-orbital ordered phase for the large onsite Coulomb interaction at 1/4 filling, and charge ordered phases for the large nearest-neighbor Coulomb interaction at $1 / 4$ and $1 / 2$ fillings. We

45 show that these staggered orders activate different types of ASOCs, which lead to different unusual properties, such as a linear magnetoelectric response and a spin splitting in the band structure. We also show that a staggered potential on the honeycomb structure gives rise to a valley splitting in the band structure at $1 / 2$ filling. Finally, we discuss the implication of our results in several candidate materials.

\section{Model and method}

We consider the model discussed in the previous studies [11, 23, 15, 22]: the two-orbital Hubbard model on the honeycomb structure under a crystalline electric field with the trigonal or trigonal prismatic symmetry. The Hamiltonian 
is given by

$$
\begin{aligned}
\mathcal{H}= & -t_{0} \sum_{\boldsymbol{k}} \sum_{m} \sum_{\sigma}\left(\gamma_{0 \boldsymbol{k}} c_{\mathrm{A} \boldsymbol{k} m \sigma}^{\dagger} c_{\mathrm{B} \boldsymbol{k} m \sigma}+\text { H.c. }\right) \\
& -t_{1} \sum_{\boldsymbol{k}} \sum_{m} \sum_{\sigma}\left(\gamma_{m \boldsymbol{k}} c_{\mathrm{A} \boldsymbol{k} m \sigma}^{\dagger} c_{\mathrm{B} \boldsymbol{k}-m \sigma}+\text { H.c. }\right) \\
& +\frac{\lambda}{2} \sum_{s} \sum_{\boldsymbol{k}} \sum_{m} \sum_{\sigma} c_{s \boldsymbol{k} m \sigma}^{\dagger}(m \sigma) c_{s \boldsymbol{k} m \sigma} \\
& +\sum_{i} \sum_{m n m^{\prime} n^{\prime}} \sum_{\sigma \sigma^{\prime}} \frac{U_{m n m^{\prime} n^{\prime}}}{2} c_{i m \sigma}^{\dagger} c_{i n \sigma^{\prime}}^{\dagger} c_{i n^{\prime} \sigma^{\prime}} c_{i m^{\prime} \sigma} \\
& +\sum_{\langle i, j\rangle} \sum_{m m^{\prime}} \sum_{\sigma \sigma^{\prime}} V n_{i m \sigma} n_{j m^{\prime} \sigma^{\prime}},
\end{aligned}
$$

where $c_{s \boldsymbol{k} m \sigma}^{\dagger}\left(c_{s \boldsymbol{k} m \sigma}\right)$ is the creation (annihilation) operator for two sublattice on the honeycomb structure $s=\mathrm{A}$ or B, wave number $\boldsymbol{k}$, orbital $m= \pm 1$, and spin $\sigma=\uparrow$ or $\downarrow ; c_{i m \sigma}^{\dagger}\left(c_{i m \sigma}\right)$ is the real-space counterpart of $c_{s \boldsymbol{k} m \sigma}^{\dagger}\left(c_{s \boldsymbol{k} m \sigma}\right)$.

The first and second terms in equation (1) represent the intra- and interorbital hoppings between nearest-neighbor sites, respectively; $\gamma_{n \boldsymbol{k}}=\sum_{i}^{3} \omega^{(i-1) n} e^{i \boldsymbol{k} \cdot \boldsymbol{\eta}_{i}}$ $(n=0, \pm 1)$, where $\omega=e^{i 2 \pi / 3}$ and $\boldsymbol{\eta}_{i}$ represents the nearest-neighbor bonds between $\mathrm{A}$ and $\mathrm{B}$ sublattices on the honeycomb structure. The third term in equation (1) represents the atomic SOC; it has a nonzero matrix element only for the diagonal $z$ component in terms of the orbital $m$, as we consider only ${ }_{65} m= \pm 1$. The fourth term stands for the onsite Coulomb interaction; we take $U_{m m m m}=U$, and $U_{m n m n}=U-2 J_{\mathrm{H}}$, and $U_{m n n m}=U_{m m n n}=J_{\mathrm{H}}(m \neq n)$, where $U$ is the onsite repulsion and $J_{\mathrm{H}}$ is the Hund's-rule coupling, respectively. The fifth term is the Coulomb interaction between nearest-neighbor sites; $n_{i m \sigma}=c_{i m \sigma}^{\dagger} c_{i m \sigma}$. We define the electron density as $n_{\mathrm{e}}=\sum_{i m \sigma}\left\langle n_{i m \sigma}\right\rangle / N$, where $N$ is the number of lattice sites; the electron filling is defined by the electron density per spin and orbital degrees of freedom in each site, namely, $n_{\mathrm{e}} / 4$.

We study the ground state of the model in equation (1) by the mean-field approximation. We adopt the Hartree-Fock approximation to decouple the onsite ${ }_{75}$ Coulomb interaction, while the nearest-neighbor Coulomb repulsion is treated by the Hartree approximation. We employ two-site unit cell, and calculate the 
mean fields by taking the sum over $64 \times 64$ grid points in the first Brillouin zone.

\section{Ground-state phase diagram}

In this section, we discuss what types of electronic orders are stabilized in the model in equation (1). We present the mean-field results at two commensurate fillings, $1 / 4\left(n_{\mathrm{e}}=1\right)$ and $1 / 2\left(n_{\mathrm{e}}=2\right)$, in sections 3.1 and 3.2 , respectively. See also [11] for the result at $1 / 4$ filling with different parameters.

\section{1. $1 / 4$ filling}

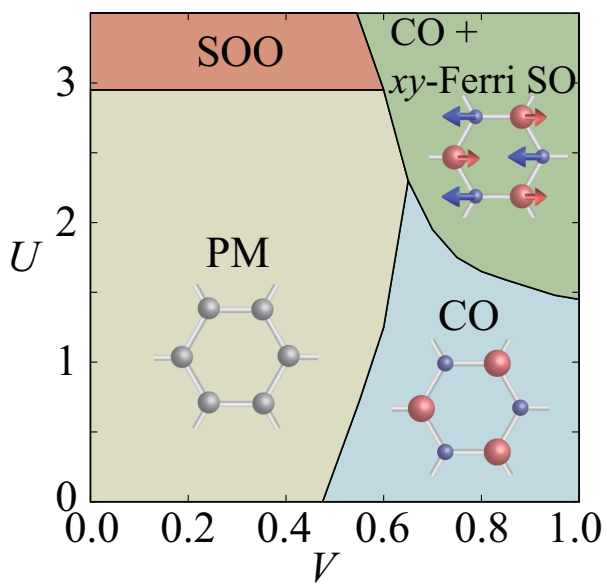

Figure 1: Ground-state phase diagram of the model in equation (1) at $1 / 4$ filling obtained by the mean-field calculations. The results are calculated for $t_{0}=t_{1}=\lambda=0.5$ and $J_{\mathrm{H}}=0.2 U$. $\mathrm{PM}, \mathrm{CO}$, SO, SOO, and Ferri represent the paramagnetic, charge ordered, spin ordered, spinorbital ordered, and ferrimagnetic states. The prefix $x y$ for the Ferri-SO denotes that the spins are polarized in the $x y$ plane. Schematic pictures for the ordering patterns except for SOO are shown. See the text for SOO in detail.

Figure 1 shows the ground-state phase diagram at $1 / 4$ filling $\left(n_{\mathrm{e}}=1\right)$. The model parameters are $t_{0}=t_{1}=\lambda=0.5$ and $J_{\mathrm{H}}=0.2 U$. As shown in the figure, the phase diagram includes four phases: paramagnetic (PM) state in the small $U$ and $V$ region, the charge ordered (CO) phase in the large $V$ region, the coexisting phase of charge order and ferrimagnetic order with the magnetic 
moments in the $x y$ plane ( $x y$-Ferri SO) in the large $U$ and $V$ region, and the spin-orbital ordered (SOO) phase in the large $U$ region. All the four phases are insulating. We note that the PM state exhibits a quantized Hall conductance $\sigma_{x y}^{\mathrm{SH}}=2[23]$.

In the $\mathrm{CO}$ state, an effective ASOC is induced, whose asymptotic form in the limit of $\lambda \rightarrow \infty$ and $k \rightarrow 0$ is given by [15]

$$
\mathcal{H}_{\mathrm{ASOC}}^{\mathrm{CO}}(\boldsymbol{k}) \propto \frac{h^{\mathrm{CO}} t_{1}^{2}}{\lambda^{2}} k_{y}\left(3 k_{x}^{2}-k_{y}^{2}\right) \rho_{0} \sigma_{0} \tau_{z} .
$$

95 
Here, $h^{\mathrm{SOO}}$ is proportional to the order parameter of the SOO state. This effective ASOC can lead to a linear magnetoelectric effect because all the symmetries on the honeycomb structure are broken [11]. Especially, the nonzero $c_{1}\left(c_{2}\right)$ induces the longitudinal (transverse) magnetoelectric effect, in which an electric polarization is induced in the parallel (perpendicular) direction to that of the magnetization.

In the phase diagram in figure 1 , the SOO phase showing the linear magnetoelectric effect is stable in the large $U$ region. This is understood from strong correlation limit as follows. Let us consider the second-order perturbation with respect to $t_{0} / U$ and $t_{0} / \lambda^{1}$. The effective Hamiltonian in the strong correlation limit can be obtained by

$$
\mathcal{H}^{\mathrm{eff}}=\mathcal{H}_{t} \frac{1}{\mathcal{H}_{U}+\mathcal{H}_{\lambda}} \mathcal{H}_{t}=\mathcal{H}^{\mathrm{eff}(0)}+\mathcal{H}^{\mathrm{eff}(1)}+\ldots,
$$

where $\mathcal{H}_{t}, \mathcal{H}_{\lambda}$, and $\mathcal{H}_{U}$ represent the first, third, and fourth terms in equation (1), respectively. Here, the right hand side of equation (4) is obtained by expanding the result in terms of $\lambda / U ; \mathcal{H}^{\text {eff }(\ell)}$ represents the effective Hamiltonian in the $\ell$-th order with respect to $\lambda$. Specifically, $\mathcal{H}^{\text {eff(0) }}$ and $\mathcal{H}^{\text {eff(1) }}$ for an $i j$ bond are obtained as

$$
\begin{aligned}
\mathcal{H}_{i j}^{\mathrm{eff}(0)}= & -\frac{4 t_{0}^{2}}{U-3 J_{\mathrm{H}}}\left(\frac{1}{4}-\boldsymbol{T}_{i} \cdot \boldsymbol{T}_{j}\right)\left(\boldsymbol{S}_{i} \cdot \boldsymbol{S}_{j}+\frac{3}{4}\right) \\
& -\frac{4 t_{0}^{2}}{U-J_{\mathrm{H}}}\left(\frac{1}{2}+2 T_{i}^{y} T_{j}^{y}\right)\left(\frac{1}{4}-\boldsymbol{S}_{i} \cdot \boldsymbol{S}_{j}\right) \\
& -\frac{4 t_{0}^{2}}{U+J_{\mathrm{H}}}\left(\frac{1}{4}+\boldsymbol{T}_{i} \cdot \boldsymbol{T}_{j}-2 T_{i}^{y} T_{j}^{y}\right)\left(\frac{1}{4}-\boldsymbol{S}_{i} \cdot \boldsymbol{S}_{j}\right), \\
\mathcal{H}_{i j}^{\mathrm{eff}(1)}= & \frac{4 t_{0}^{2} J_{\mathrm{H}}^{2} \lambda}{\left(U-J_{\mathrm{H}}\right)^{2}\left(U-3 J_{\mathrm{H}}\right)^{2}}\left[\mathcal{P}_{T_{i}}^{+} \mathcal{P}_{T_{j}}^{-} \mathcal{P}_{S_{i}}^{-} \mathcal{P}_{S_{j}}^{+}+\mathcal{P}_{T_{i}}^{-} \mathcal{P}_{T_{j}}^{+} \mathcal{P}_{S_{i}}^{+} \mathcal{P}_{S_{j}}^{-}\right. \\
& \left.-\left(T_{i}^{-} T_{j}^{+} S_{i}^{+} S_{j}^{-}+T_{i}^{+} T_{j}^{-} S_{i}^{-} S_{j}^{+}\right)\right],
\end{aligned}
$$

respectively. Here, $\boldsymbol{S}_{i}$ and $\boldsymbol{T}_{i}$ are the spin and orbital operators, which are

\footnotetext{
${ }^{1}$ In this analysis, we ignore $t_{1}$ as the SOO phase remains stable for small $t_{1}$. We also omit the nearest-neighbor repulsion $V$.
} 
represented by

$$
\boldsymbol{S}_{i}=\frac{1}{2} \sum_{m} \sum_{\sigma \sigma^{\prime}} c_{i m \sigma}^{\dagger} \boldsymbol{\sigma}_{\sigma \sigma^{\prime}} c_{i m \sigma^{\prime}}, \quad \boldsymbol{T}_{i}=\frac{1}{2} \sum_{m m^{\prime}} \sum_{\sigma} c_{i m \sigma}^{\dagger} \boldsymbol{\tau}_{m m^{\prime}} c_{i m^{\prime} \sigma},
$$

respectively. In equation (6), $\mathcal{P}_{\Lambda_{i}}^{ \pm}(\Lambda=T$ or $S)$ are the projection operators defined as $\mathcal{P}_{\Lambda_{i}}^{ \pm}=\frac{1}{2} \pm \Lambda_{i}^{z}$. $\mathcal{H}_{i j}^{\text {eff }(0)}$ describes the spin-orbital exchange interactions, similar to that for the twofold-degenerate $e_{g}$ orbitals, where the SOC is irrelevant [25]. On the other hand, $\mathcal{H}_{i j}^{\mathrm{eff}(1)}$, which is the leading contribution from the SOC, includes characteristic terms, $T_{i}^{-} T_{j}^{+} S_{i}^{+} S_{j}^{-}+T_{i}^{+} T_{j}^{-} S_{i}^{-} S_{j}^{+}$. These off-diagonal components favor the spin-orbital orders of SOO type. Indeed, for a two-site problem, $\mathcal{H}_{i j}^{\text {eff(1) }}$ has the lowest energy for the state $(1 / \sqrt{2})\left(c_{\mathrm{A}-1 \uparrow}^{\dagger} c_{\mathrm{B}+1 \downarrow}^{\dagger}-\right.$ $\left.c_{\mathrm{A}+1 \downarrow}^{\dagger} c_{\mathrm{B}-1 \uparrow}^{\dagger}\right)|0\rangle$, where $|0\rangle$ is the vacuum. This state is a constituent of the SOO discussed above. Thus, the analysis in the strong correlation limit explains the stability of the SOO phase in the large $U$ limit.

\section{2. $1 / 2$ filling}

(a)

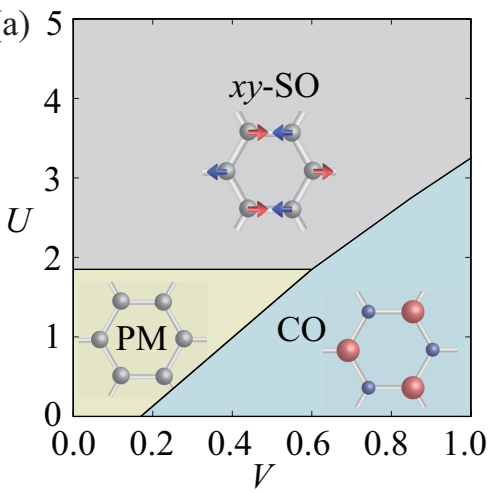

(b)

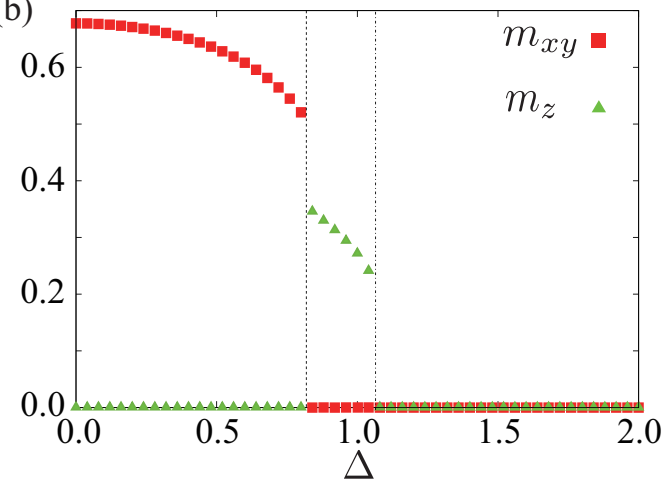

Figure 2: (a) Ground-state phase diagram of the model in equations (1) at $1 / 2$ filling obtained by the mean-field calculations. The results are calculated for $t_{0}=t_{1}=\lambda=0.5$ and $J_{\mathrm{H}}=0.1 U$. Schematic picture for the ordering pattern is shown for each phase. (b) Inplane and out-of-plane magnetic moments $m_{x y}$ and $m_{z}$, respectively, as functions of the staggered potential $\Delta$ in equation (8) at $U=3$ and $V=0.5$.

Figure 2(a) shows the ground-state phase diagram for the model in equation (1) at $1 / 2$ filling $\left(n_{\mathrm{e}}=2\right)$ obtained by the mean-field calculation for 
$t_{0}=t_{1}=\lambda=0.5$ and $J_{\mathrm{H}}=0.1 U$. There are three phases: the PM phase in the small $U$ and $V$ region, the CO phase in the large $V$ region, and the staggered magnetic ordered phase with the $x y$-spin component $(x y$-SO) in the large $U$ region. All the three phases are insulating. As in the $1 / 4$ filling case, the PM state is a topological insulator, where the spin Hall conductivity is quantized at $\sigma_{x y}^{\mathrm{SH}}=2$ [23]. As increasing $V$, the PM state changes into the CO state, which shows the antisymmetric spin splitting in the band structure, similar to the $\mathrm{CO}$ state at $1 / 4$ filling.

The $x y$-SO phase in the large $U$ region is characterized by the spin order parameter given by $c_{1}\left\langle\sigma_{x}\right\rangle+c_{2}\left\langle\sigma_{y}\right\rangle$, where $-1 \leq c_{1} \leq 1$ and $-1 \leq c_{2} \leq 1$. Unfortunately, the $x y$-SO phase does not show peculiar electronic structure, magnetotransport, and magnetoelectric phenomena within the linear response theory. However, an interesting band structure appears when the magnetic moment acquires the $z$ component. Although this state is not obtained in the parameter space we studied, we find that it is stabilized by introducing an additional symmetry-breaking field in the charge sector given by

$$
\mathcal{H}_{\mathrm{pot}}=\Delta \sum_{s \boldsymbol{k} m \sigma}\left(\rho_{z}\right)_{s s} c_{s \boldsymbol{k} m \sigma}^{\dagger} c_{s \boldsymbol{k} m \sigma},
$$

where $\Delta$ denotes the magnitude of the staggered potential. Figure 2(b) shows the $\Delta$ dependence of the magnetic order parameters $m_{x y}=\sqrt{m_{x}^{2}+m_{y}^{2}}$ and $m_{z}$ for $U=3$ and $V=0.5$, where $m_{\mu}$ is the magnetization per site in the $\mu$ direction $(\mu=x, y, z)$. The $m_{x y}$ component is suppressed as $\Delta$ increases and becomes zero for $\Delta \gtrsim 0.8$. Instead, the $m_{z}$ component becomes nonzero in the intermediate $\Delta$ region for $0.8 \lesssim \Delta \lesssim 1.1$. For $\Delta \gtrsim 1.1$, the system loses both spin and orbital orders, just leaving a charge disproportionation (CD) by the staggered potential.

We show the band structures for the three phases in figure 3 . The staggered magnetic ordering with the $z$-spin component $(z$-SO) in the intermediate $\Delta$ region induces a valley splitting in the band structure, as shown in figure 3(b) [18], while only a spin splitting due to the staggered potential in equation (8) is observed in the $x y$-SO state in the small $\Delta$ region [figure $3(\mathrm{a})]$ and the PM state 
in the large $\Delta$ region [figure 3(c)].
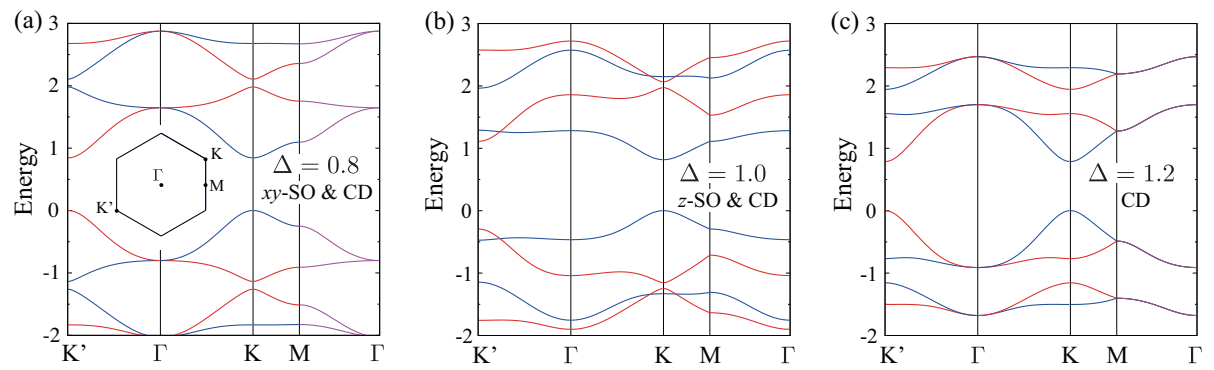

Figure 3: Electronic band structures for (a) $x y$-SO, (b) $z$-SO, and (c) PM states at $\Delta=0.8$, 1.0, and 1.2, respectively. CD represents the charge disproportionation due to the staggered potential. The blue (red) curves show the bands with up(down)-spin polarization in the $z$ direction. In the inset of (a), the first Brillouin zone is shown.

\section{Summary and concluding remarks}

We have investigated the stability of electronic orders which spontaneously break the spatial inversion symmetry for a minimal two-band model on the honeycomb structure. By studying the ground state of the model by the mean-field approximation, we have found several insulating phases, such as the CO states at $1 / 4$ and $1 / 2$ fillings and the SOO state at $1 / 4$ filling. The obtained staggered phases activate different type of effective ASOCs by breaking of spatial inversion symmetry, which gives rise to the fascinating physical properties, such as the spin splitting in the band structure in the $\mathrm{CO}$ state and linear magnetoelectric effects in the SOO state. In addition, we have investigated the stability of the SOO state in the strong correlation limit. We have also shown that the staggered potential can switch the magnetic ordered phases, and results in another type of effective ASOC leading to a valley splitting in the band structure.

Let us comment on relevant materials for future studies. There are several candidate materials with the honeycomb structures from $d$ electron systems to $f$ electron systems, such as trichalcogenides $M X^{\prime} X_{3}$ ( $M$ : transition metal, $X$ : chalcogen, $\left.X^{\prime}=\mathrm{P}, \mathrm{Si}, \mathrm{Ge}\right)[26,27]$ and a Yb-based heavy-fermion compound 
$\beta-\mathrm{YbAlB}_{4}[28,29,30]$. From a symmetry point of view, it is expected that similar ASOC physics is realized for these materials once a staggered electronic order occurs. Especially, the interesting SOO state, which shows linear magnetoelectric effects, may be achieved when the electron correlations and the SOC are sufficiently large.

An extension of our results to noncentrosymmetric systems is also an interesting future problem. As demonstrated in the last part of Sec. 3.2, the staggered potential, which breaks the spatial inversion symmetry, leads to the spin splitting in the band structure. As an effect of the two different atoms in the different sublattices can be mimicked by the staggered potential, this situation corresponds to monolayer transition metal dichalcogenides $M X_{2}$ with $2 \mathrm{H}$ structure $[31,32,33,34]$ where the alignment of the transition metal $M$ and the chalcogen $X$ forms the honeycomb structure. Indeed, a similar spin splitting in the band structure is observed in the monolayer $2 \mathrm{H}-M X_{2}$ [35]. Our results suggest that a valley splitting is expected in addition to the spin splitting once the $z$-SO is stabilized for a sufficiently large staggered potential in the monolayer $2 \mathrm{H}-\mathrm{MX}_{2}$ [18]. Further studies from both theoretical and experimental sides are desired for such exploration.

\section{Acknowledgments}

This research was supported by JSPJ KAKENHI Grants Numbers 15K05176, 15H05885 (J-Physics), and 16H06590. Parts of the numerical calculations were performed in the supercomputing systems in ISSP, the University of Tokyo.

\section{References}

[1] E. Bauer, M. Sigrist (Eds.), Non-Centrosymmetric Superconductors: Introduction and Overview (Lecture Notes in Physics), 2012th Edition, Springer, 2012.

[2] M. Z. Hasan, C. L. Kane, Colloquium : Topological insulators, Rev. Mod. Phys. 82 (2010) 3045-3067. 
[3] X.-L. Qi, S.-C. Zhang, Topological insulators and superconductors, Rev. Mod. Phys. 83 (2011) 1057-1110.

[4] D. Khomskii, Classifying multiferroics: Mechanisms and effects, Physics 2 (2009) 20.

[5] J. E. Hirsch, Spin hall effect, Phys. Rev. Lett. 83 (1999) 1834-1837.

[6] J. Sinova, D. Culcer, Q. Niu, N. A. Sinitsyn, T. Jungwirth, A. H. MacDonald, Universal intrinsic spin hall effect, Phys. Rev. Lett. 92 (2004) 126603.

[7] M. Fiebig, Revival of the magnetoelectric effect, J. Phys. D: Appl. Phys. 38 (8) (2005) R123.

[8] Y. Yanase, Magneto-electric effect in three-dimensional coupled zigzag chains, J. Phys. Soc. Jpn. 83 (1) (2014) 014703.

[9] S. Hayami, H. Kusunose, Y. Motome, Toroidal order in metals without local inversion symmetry, Phys. Rev. B 90 (2014) 024432.

[10] V. Sakhnenko, N. Ter-Oganessian, The magnetoelectric effect due to local noncentrosymmetry, J. Phys.: Condens. Matter 24 (26) (2012) 266002.

[11] S. Hayami, H. Kusunose, Y. Motome, Spontaneous parity breaking in spinorbital coupled systems, Phys. Rev. B 90 (2014) 081115.

[12] T. Hitomi, Y. Yanase, Electric octupole order in bilayer ruthenate sr3ru2o7, J. Phys. Soc. Jpn. 83 (11) (2014) 114704.

[13] S. Hayami, H. Kusunose, Y. Motome, Spontaneous multipole ordering by local parity mixing, J. Phys. Soc. Jpn. 84 (6) (2015) 064717.

[14] S. Sumita, Y. Yanase, Superconductivity in magnetic multipole states, Phys. Rev. B 93 (2016) 224507.

245 [15] S. Hayami, H. Kusunose, Y. Motome, Emergent spin-valley-orbital physics by spontaneous parity breaking, J. Phys.: Condens. Matter 28 (39) (2016) 395601. 
[16] Y. Sugita, Y. Motome, Topological insulators from electronic superstructures, J. Phys. Soc. Jpn. 85 (7) (2016) 073709.

[17] B. Volkov, A. Gorbatsevich, Y. V. Kopaev, V. Tugushev, Macroscopic current states in crystals, Zh. Eksp. Teor. Fiz 81 (1981) 742.

[18] X. Li, T. Cao, Q. Niu, J. Shi, J. Feng, Coupling the valley degree of freedom to antiferromagnetic order, Proc. Natl. Acad. Sci. U.S.A. 110 (10) (2013) $3738-3742$.

[19] S. Hayami, H. Kusunose, Y. Motome, Asymmetric magnon excitation by spontaneous toroidal ordering, J. Phys. Soc. Jpn. 85 (5) (2016) 053705.

[20] G. Gitgeatpong, M. Suewattana, S. Zhang, A. Miyake, M. Tokunaga, P. Chanlert, N. Kurita, H. Tanaka, T. J. Sato, Y. Zhao, K. Matan, Highfield magnetization and magnetic phase diagram of $\alpha-\mathrm{cu}_{2} \mathrm{v}_{2} \mathrm{O}_{7}$, Phys. Rev. B 95 (2017) 245119.

[21] G. Gitgeatpong, Y. Zhao, P. Piyawongwatthana, Y. Qiu, L. W. Harriger, N. P. Butch, T. J. Sato, K. Matan, Nonreciprocal magnons and symmetrybreaking in the noncentrosymmetric antiferromagnet, Phys. Rev. Lett. 119 (2017) 047201.

[22] Y. Yanagi, H. Kusunose, Optical selection rules in spin-orbit coupled systems on honeycomb lattice, J. Phys. Soc. Jpn. 86 (8) (2017) 083703.

[23] S. Hayami, H. Kusunose, Y. Motome, Quantum spin hall effect in a twoorbital model on a honeycomb lattice, J. Phys.: Conf. Ser. 592 (1) (2015) 012131.

[24] E. I. Rashba, Properties of semiconductors with an extremum loop. 1. cyclotron and combinational resonance in a magnetic field perpendicular to the plane of the loop, Sov. Phys. Solid State 2 (6) (1960) 1109-1122.

[25] K. I. Kugel, D. Khomskii, The jahn-teller effect and magnetism: transition metal compounds, Phys. Usp. 25 (4) (1982) 231-256. 
[31] S. Jobic, P. Deniard, R. Brec, J. Rouxel, M. Drew, W. David, Properties of the transition metal dichalcogenides: The case of irs 2 and irse 2, J. Solid State Chem. 89 (2) (1990) 315-327.

[32] R. Clarke, E. Marseglia, H. Hughes, A low-temperature structural phase

[33] M. N. Ali, J. Xiong, S. Flynn, J. Tao, Q. D. Gibson, L. M. Schoop, T. Liang, N. Haldolaarachchige, M. Hirschberger, N. Ong, R. J. Cava, Large, nonsaturating magnetoresistance in wte2, Nature 514 (7521) (2014) 205-208.

[34] X. Qian, J. Liu, L. Fu, J. Li, Quantum spin hall effect in two-dimensional 
[35] D. Xiao, W. Yao, Q. Niu, Valley-contrasting physics in graphene: Magnetic moment and topological transport, Phys. Rev. Lett. 99 (2007) 236809. 